\title{
A CANCELLATION CRITERION FOR FINITE-RANK TORSION-FREE ABELIAN GROUPS
}

\author{
J. STELZER
}

\begin{abstract}
In this paper, a necessary ring-theoretical criterion is given for a finite-rank torsion-free abelian group to have the cancellation property. This generalizes results obtained by L. Fuchs and F. Loonstra [5] for the rank-one case and resolves the cancellation problem for strongly indecomposable groups.
\end{abstract}

Introduction. An abelian group $G$ has the cancellation property if for any two abelian groups $H$ and $K, G \oplus H \cong G \oplus K$ implies that $H \cong K$. Examples for the failure of cancellation are abundant, even among torsion-free abelian groups of finite rank; B. Jonsson [6, 7]. On the other hand, the cancellation property does hold for finitely generated abelian groups (E. A. Walker [8]), and the torsion-free rank-one groups that satisfy cancellation have been completely determined by $\mathrm{L}$. Fuchs and F. Loonstra [5].

In this note, and in a subsequent paper, these fragmentary results will be systematized. Here we establish a necessary cancellation criterion for torsion-free abelian groups of finite rank.

THEOREM A. Let $G$ be a torsion-free abelian group of finite rank satisfying the cancellation property. Write $G=B \oplus C$, where $B$ is free and $C$ contains no free summand. Then for each $n \in Z \backslash\{0\}$, every unit of $E(C) / n E(C)$ lifts to a unit of $E(C)$.

The question arises of whether or not the above criterion is also sufficient. To this end, the cancellation property will be studied in the context of two related notions, substitution and stable range. An abelian group $G$ has the substitution property if for any abelian group $A=G_{1} \oplus H=G_{2} \oplus K$ and $G_{1} \cong G_{2} \subseteq G$, there exists a $G_{3} \subseteq A$ such that $A=G_{3} \oplus H=G_{3} \oplus K$. A ring $R$ has 1 in the stable range if, for any $f_{1}, g_{1}, f_{2}, g_{2} \in R$ with $f_{1} g_{1}+f_{2} g_{2}=1$, there exists an $h \in R$ such that $f_{1}+f_{2} h$ is a unit of $R$.

Clearly, substitution implies cancellation. The connection between substitution and stable range properties, which holds in a more general setting, is given by $\mathrm{R}$. B.

Received by the editors February 16, 1984.

1980 Mathematics Subject Classification. Primary 20K15.

Key words and phrases. Finite-rank torsion-free abelian groups, cancellation property.

${ }^{1}$ The results of this paper are contained in the author's New Mexico State University dissertation, which was supervised by Dr. D. M. Arnold. 
Warfield [9]: An abelian group $G$ has the substitution property if and only if 1 is in the stable range of $E(G)$, the endomorphism ring of $G$.

With these results, it is easy to settle the cancellation problem in the strongly indecomposable case.

THEOREM B. Let $G$ be a strongly indecomposable torsion-free abelian group of finite rank. Then the following are equivalent:

(1) $G$ has the cancellation property.

(2) Either $G \cong Z$, or, for each $n \in Z \backslash\{0\}$, every unit of $E(G) / n E(G)$ lifts to a unit of $E(G)$.

(3) Either $G \cong Z$, or 1 is in the stable range of $E(G)$.

(4) Either $G \cong Z$, or $G$ has the substitution property.

Theorem B will be generalized substantially in a subsequent paper. In view of Theorem $\mathrm{A}$, this amounts to resolving a ring-theoretical problem: Let $R$ be a ring with finite-rank torsion-free additive group. Suppose that, for each $n \in Z \backslash\{0\}$, every unit of $R / n R$ lifts to a unit of $R$. Does $R$ have 1 in the stable range? In the general case, more complicated techniques from ring theory and algebraic number theory are required than for the proof of Theorem B.

Proof of the theorems. The terminology is as in L. Fuchs [2, 3] and D. M. Arnold [1]. Unless otherwise specified, tensor products are taken with respect to $Z$. The set of all primes of $Z$ is denoted by $\pi$. If $p \in \pi$, define

$$
\begin{aligned}
& Z_{p}=\text { localization of } Z \text { with respect to } p, \\
& Z_{p}^{*}=\text { ring of } p \text {-adic integers, } \\
& K_{p}=\text { field of } p \text {-adic numbers. }
\end{aligned}
$$

For a torsion-free abelian group $G$ of finite rank, set

$$
G_{p}=Z_{p} \otimes G, \quad \hat{G}_{p}=p \text {-adic completion of } G .
$$

Finally, if $P$ is a subset of $\pi$, write

$$
\begin{aligned}
\bar{P} & =\pi \backslash P, \\
Z(P) & =\left\langle p^{-k}: p \in \bar{P}, k \in N\right\rangle, \quad \text { a subgroup of } Q, \\
G(P) & =Z(P) \otimes G .
\end{aligned}
$$

The proof of Theorem A is based on a lemma established by L. Fuchs and F. Loonstra [5] in deriving cancellation criteria for subgroups of $Q$.

LEMMA 1. Let $G$ be a torsion-free abelian group satisfying the cancellation property. Assume that there exists a torsion-free abelian group $A$ such that:

(1) $E(A) \cong Z$.

(2) For each $n \in Z \backslash\{0\}$, there is an epimorphism $\beta: A \rightarrow G / n G$.

(3) $\operatorname{Hom}(G, A)=\operatorname{Hom}(A, G)=0$.

Then for each $n \in Z \backslash\{0\}$, every unit of $E(G) / n E(G)$ lifts to unit of $E(G)$. 
REMARK 1. In [4], L. Fuchs states this theorem without imposing any requirements on $E(G)$; but his proof refers to a lemma which is incorrect. A corrected version is given by D. M. Arnold [1, p. 94, Lemma 8.10]. Although he only deals with the finite-rank case, his arguments hold for arbitrary torsion-free groups, under the convention that a unit is a two-sided unit.

REMARK 2. If $G$ is of finite rank and contains no free summands, then a group $A$, complying with (1), (2), and $\operatorname{Hom}(G, A)=0$ exists by L. Fuchs [3, Example 5, p. 125] or D. M. Arnold [1, Example 2.7, p. 26]. These examples will be modified to guarantee that $\operatorname{Hom}(A, G)=0$.

Lemma 2. Let $t \in N, t \geqslant 2$, and let $P$ be a finite set of primes. For each $p \in P$, let $\sigma_{2}(p), \ldots, \sigma_{t}(p)$ be arbitrary units of $Z_{p}^{*}$, algebraically independent over $Q$. Then there is a torsion-free abelian group $A$, depending on these p-adic integers, which satisfies the following properties:

(1') For each $p \in P, E\left(A_{p}\right) \cong Z_{p}$.

(1) $E(A) \cong Z$.

(2) For each $e \in N$,

$$
A / p^{e} A \cong \begin{cases}\left(Z / p^{e} Z\right)^{t-1} & \text { if } p \in P, \\ \left(Z / p^{e} Z\right)^{t} & \text { if } p \in \pi \backslash P .\end{cases}
$$

(3) If $B$ is a subgroup of $A$ with rank $B \leqslant t-1$, then $B$ is free.

Proof. Each $\sigma_{j}(p)$ can be written as

$$
\sigma_{j}(p)=\sum_{e=0}^{\infty} s_{j}(p, e) p^{e} .
$$

where each $s_{j}(p, e)$ is an integer with $0 \leqslant s_{j}(p, e)<p$. Set

$$
\sigma_{j}(p, e)=s_{j}(p, 0)+s_{j}(p, 1) p+\cdots+s_{j}(p, e-1) p^{e-1}
$$

and define

$$
\begin{aligned}
F & =Z z_{1} \oplus Z z_{2} \oplus \cdots \oplus Z z_{t}, \\
A & =\left\langle z_{1}, \ldots, z_{t} ; \bar{z}(p, e): p \in P, e \in N\right\rangle \subseteq Q F,
\end{aligned}
$$

where $\bar{z}(p, e)=p^{-e}\left[z_{1}+\sigma_{2}(p, e) z_{2}+\cdots+\sigma_{t}(p, e) z_{t}\right]$.

$\left(1^{\prime}\right)$ Imitate the proof of Example 5 in L. Fuchs [3, p. 125].

(1) Let $\phi \in E(A)$. For all $p \in P$, the extension of $\phi$ to an endomorphism of $A_{p}$ acts like multiplication by a number belonging to $Z_{p}$. Hence $\phi$ itself is a multiplication by some $c \in Z(P)$. Since $A_{p}=F_{p}$ for $p \in P$, it follows that $c \in Z(P) \cap Z(\bar{P})$ $=Z$. Therefore, $E(A) \cong Z$.

(2) For $p \in \bar{P}$, we have $A / p^{e} A \cong A_{p} / p^{e} A_{p}=F_{p} / p^{e} F_{p}=\left(Z / p^{e} Z\right)^{t}$. If $p \in P$, the group $A_{p} / F_{p}$ is generated by the elements $\bar{z}(p, e)+F_{p}, e \in N$, with the relations $p \bar{z}(p, e+1)+F_{p}=\bar{z}(p, e)+F_{p}$. Let $T=A / F$ so that $T_{p}=(A / F)_{p}=A_{p} / F_{p} \cong$ $Z\left(p^{\infty}\right)$ and $T \cong \oplus_{p \in P} Z\left(p^{\infty}\right)$. By a well-known theorem on $p$-ranks, D. M. Arnold [1, Theorem 0.2, p. 3],

$$
\operatorname{dim} T[p]+p \text {-rank } A=p \text {-rank } F+p-\operatorname{rank} T ;
$$


hence $1+p$-rank $A=p$-rank $F$ and $p$-rank $A=t-1$. Consequently $A / p^{e} A \cong$ $\left(Z / p^{e} Z\right)^{t-1}$ for each $p \in P, e \in N$.

(3) Without loss of generality, assume that $B$ is pure in $A$. The map $\phi: B / B \cap F$ $\rightarrow A / F, b+(B \cap F) \mapsto b+F$ is an embedding with image $(B+F) / F \subseteq A / F \cong$ $\oplus_{p \in P} Z\left(p^{\infty}\right)$. If $B / B \cap F$ is finite, $B \cong B \cap F$ is free. So it remains to exclude the case that $B / B \cap F$ is infinite. In this situation, $B / B \cap F \cong(B+F) / F$ would have to be $p$-divisible by some $p \in P$, since $P$ is finite. This would imply that $\left(B_{p}+F_{p}\right) / F_{p}=A_{p} / F_{p}$ and $A_{p}=B_{p}+F_{p}$. Consequently, $A_{p} / B_{p} \cong F_{p} /\left(F_{p} \cap B_{p}\right)$ would be free over the ring $Z_{p}$; in particular the exact sequence

$$
0 \rightarrow B_{p} \rightarrow A_{p} \rightarrow A_{p} / B_{p} \rightarrow 0
$$

would split. This is impossible, since $E\left(A_{p}\right) \cong Z_{p}$ by $\left(1^{\prime}\right)$.

Lemma 3. Let $t \in N, t \geqslant 2$, and let $P$ be a finite set of primes. For each $p \in P$, let $R(p)$ be an arbitrary reduced p-local group with rank $R(p) \leqslant t-1$. Then there exist $p$-adic integers $\sigma_{2}(p), \ldots, \sigma_{t}(p)$ such that the group A constructed in Lemma 2 satisfies $\operatorname{Hom}(A, R(p))=0$ for each $p \in P$.

Proof. First fix $p \in P$ and define $\sigma_{2}(p), \ldots, \sigma_{t}(p)$. In order to simplify notation, the $p$ is omitted by replacing $R(p)$ with $R$ and $\sigma_{j}(p)$ with $\sigma_{j}$.

Let $x_{1}, \ldots, x_{m}$ be a maximal linearly independent subset of $R$. The $p$-adic completion $\hat{R}$ of $R$ has a basis over $Z_{p}^{*}$, say

$$
\hat{R}=Z_{p}^{*} y_{1} \oplus \cdots \oplus Z_{p}^{*} y_{n} .
$$

Identifying $R$ as a $Z_{p}$-submodule of $\hat{R}$, the elements $x_{k}$ are represented by

$$
x_{k}=\sum_{l=1}^{n} \rho_{k l} y_{l}, \rho_{k l} \in Z_{p}^{*}
$$

Choose $\sigma_{2}=\sigma_{2}(p), \ldots, \sigma_{t}=\sigma_{t}(p) \in Z_{p}^{*} \backslash p Z_{p}^{*}$, algebraically independent over

$$
Q\left(\rho_{k l} ; k=1, \ldots, m ; l=1, \ldots, n\right) \subseteq K_{p}
$$

and form the group $A$ according to Lemma 2 .

It remains to show that $\operatorname{Hom}(A, R(p))=0$ for each $p \in P$. Again, write $R=$ $R(p), \sigma_{j}=\sigma_{j}(p)$ and $\sigma_{j}(e)=\sigma_{j}(p, e)$. Each homomorphism $\phi: A \rightarrow R$ is determined by the images of $z_{1}, \ldots, z_{i}$; there is some $a \in Z$ with $a \phi: A \rightarrow R$ and

$$
z_{j}^{a \phi}=\sum_{k=1}^{m} \alpha_{j k} x_{k}
$$

where $\alpha_{j k} \in Z_{p}$. It may be assumed that $a=1$, since $a \phi=0$ forces $\phi=0$. By the definition of $A$,

$$
p^{e}\left|\left[z_{1}+\sigma_{2}(e) z_{2}+\cdots+\sigma_{t}(e) z_{t}\right]^{\phi}\right|
$$

so

$$
p^{e}\left|\sum_{k=1}^{m}\left[\alpha_{1 k}+\sum_{j=2}^{t} \sigma_{j}(e) \alpha_{j k}\right]\right| x_{k},
$$


where divisibility holds in $R$. Hence the right side converges to 0 as $e \rightarrow \infty$. Substituting (I), we obtain

$$
\sum_{k=1}^{m} \sum_{l=1}^{n}\left(\alpha_{1 k}+\sum_{j=2}^{t} \sigma_{j} \alpha_{j k}\right) \rho_{k l} y_{l}=0
$$

and

$$
\sum_{k=1}^{m} \alpha_{1 k} \rho_{k l}+\sum_{j=2}^{t}\left(\sum_{k=1}^{m} \alpha_{j k} \rho_{k l}\right) \sigma_{j}=0 \quad(l=1, \ldots, n) .
$$

The algebraic independence of the $\sigma_{j}$ yields

$$
\sum_{k=1}^{m} \alpha_{j k} \rho_{k l}=0(j=1, \ldots, t ; l=1, \ldots, n) .
$$

Multiplying on the right by $y_{l}$ and summing up over $l$, we get

$$
\begin{aligned}
0 & =\sum_{l=1}^{n} \sum_{k=1}^{m} \alpha_{j k} \rho_{k l} y_{l} \\
& =\sum_{k=1}^{m} \alpha_{j k} x_{k}=z_{j}^{\phi}(j=1, \ldots, t) .
\end{aligned}
$$

Thus $\phi=0$, as desired.

THEOREM. Let $G$ be a torsion-free abelian group of finite rank, say rank $G=t-1$, and assume that $G$ contains neither a free nor a divisible summand. Then there exists $a$ torsion-free group $A$ with rank $A=t$ satisfying:

(1) $E(A) \cong Z$.

(2) For each $n \in Z \backslash\{0\}$, there is an epimorphism $\beta: A \rightarrow G / n G$.

(3) Each subgroup of $A$ of rank $\leqslant t-1$ is free.

(4) $\operatorname{Hom}(G, A)=\operatorname{Hom}(A, G)=0$.

Proof. For a set $P$ of primes, let $D(P)$ denote the $p$-divisible part of $G$, i.e. $D(P)=\left(g \in G: p^{e} \mid g\right.$ for all $\left.p \in P, e \in N\right)$. The first goal is to find finitely many primes $p_{1}, \ldots, p_{m}$, associated with sets $P_{0}=\varnothing, P_{1}=\left\{p_{1}\right\}, \ldots, P_{m}=\left\{p_{1}, \ldots, p_{m}\right\}$, and groups $R\left(p_{1}\right), \ldots, R\left(p_{m}\right)$, such that the following conditions hold:

(1') For all $k \in\{1 ; \ldots ; m\}, R\left(p_{k}\right)$ is $p_{k}$-local, reduced and with rank $\geqslant 1$.

(2') If $0 \leqslant k \leqslant m-1$,

$$
Z\left(P_{k+1}\right) \otimes D\left(P_{k}\right)=R\left(p_{k+1}\right) \oplus\left[Z\left(P_{k+1}\right) \otimes D\left(P_{k+1}\right)\right] .
$$

(3') $D\left(P_{m}\right)=0$.

The construction is by induction. To begin with, set $P_{0}=\varnothing$ and $D\left(P_{0}\right)=G$. Now assume that $p_{1}, \ldots, p_{k}$, as well as the corresponding groups $R\left(p_{1}\right), \ldots, R\left(p_{k}\right)$ have been found. If $D\left(P_{k}\right)=0$, we are done. Otherwise, there is a prime $p_{k+1} \notin P_{k}$ such that $D\left(P_{k}\right)$ is not divisible by $p_{k+1}$, since $G$ contains no divisible subgroup. With $P_{k+1}=P_{k} \cup\left\{p_{k+1}\right\}$, the group $D\left(P_{k+1}\right)$ induces a decomposition $Z\left(P_{k+1}\right) \otimes$ $D\left(P_{k}\right)=R\left(p_{k+1}\right) \oplus\left[Z\left(P_{k+1}\right) \otimes D\left(P_{k+1}\right)\right]$ where $R\left(p_{k+1}\right)$ is $p_{k+1}$-local, reduced, and of rank $\geqslant 1$. Since the group $G$ has finite rank and rank $D\left(P_{k+1}\right)<\operatorname{rank} D\left(P_{k}\right)$ for all $k$, there exists an $m \in N$ such that $D\left(P_{m}\right)=0$. 
Construct $A$ according to Lemma 3, with respect to the groups $R\left(p_{k}\right), k \in$ $\{1 ; \ldots ; m\}$. The assertions (1) and (3) hold by Lemma 2. Assertion (2) is an immediate consequence, since $G / p^{e} G$ is isomorphic to a subgroup of $\left(Z / p^{e} Z\right)^{t-1}$. Furthermore, Lemma 2 shows that $\operatorname{Hom}(G, A)=0: G$ has no free summands, and rank $G=t-1$. On the other hand, if $\phi: A \rightarrow G$ is a homomorphism, then by the construction of $A, A^{\phi} \subseteq\left[Z\left(P_{1}\right) \otimes D\left(P_{1}\right)\right] \cap G=D\left(P_{1}\right)$. Induction yields $A^{\phi} \subseteq$ $D\left(P_{k}\right), k=1, \ldots, m$. So $A^{\phi}=0$, as desired.

Proof of Theorem A. Since the cancellation property is preserved by direct summands, it is valid for $C$. The group $C$ can be expressed as a direct sum of a divisible group $D$ and a reduced group $H: C=D \oplus H$. The group $H$ has cancellation and satisfies the hypothesis of the above theorem. Lemma 1 implies that for each $n \in Z \backslash\{0\}$, every unit of $E(H) / n E(H)$ lifts to a unit of $E(H)$, and a routine calculation shows that this lifting property extends to $E(C)$.

Proof of TheOREM B. By Theorem A and the introductory remarks, it only needs to be shown that (2) implies (3). But this follows from D. M. Arnold [1, Corollary 8.5.b, p. 92].

\section{REFERENCES}

1. D. M. Arnold, Finite rank torsion free abelian groups and rings, Lecture Notes in Math., vol. 931, Springer-Verlag, Berlin, Heidelberg and New York, 1982.

2. L. Fuchs, Infinite abelian groups, vol. I, Academic Press, New York, 1970.

3. Infinite abelian groups, vol. II, Academic Press, New York, 1973.

4. The cancellation property for modules, Lecture Notes in Math., vol. 246, Springer-Verlag, Berlin, Heidelberg and New York, 1970.

5. L. Fuchs and F. Loonstra, On the cancellation of modules in direct sums over Dedekind domains, Indag. Math. 33 (1971), 163-169.

6. B. Jonsson, On direct decompositions of torsion-free abelian groups, Math. Scand. 5 (1957), 230-235.

7. On direct decompositions of torsion-free abelian groups, Math. Scand. 7 (1959), 361-371.

8. E. A. Walker, Cancellation in direct sums of groups, Proc. Amer. Math. Soc. 7 (1956), 898-902.

9. R. B. Warfield, Jr., Cancellation of modules and groups and stable range of endomorphism rings, Pacific J. Math. 91 (1980), 457-485.

College of Engineering and Applied Science, University of Colorado, Colorado Springs, COLORADO 80933

Curent address: Siemens AG, E STE 31, Günther-Scharowski-Str. 2, 8520 Erlangen, West Germany 